\title{
Dynamical problem of micropolar viscoelasticity
}

\author{
Rajneesh Kumar and Suman Choudhary \\ Mathematics Department, Kurukshetra University, Kurukshetra 136 119, Haryana, India.
}

The dynamic problem in micropolar viscoelastic medium has been investigated by employing eigen value approach after applying Laplace and Fourier transformations. An example of infinite space with concentrated force at the origin has been presented to illustrate the application of the approach. The integral transforms have been inverted by using a numerical technique to obtain the displacement components, force stresses, couple stress and microrotation in the physical domain. The results for these quantities are given and illustrated graphically.

\section{Introduction}

Modern engineering structures are often made up of materials possessing an internal structure. Polycrystalline materials, materials with fibrous or coarse grain structure come in this category. Classical elasticity is inadequate to represent the behaviour of such materials. The analysis of such materials requires incorporating the theory of oriented media. For this reason, micropolar theories were developed by Eringen (1966a,b; 1976) for elastic solids, fluids and further for non-local polar fields and are now universally accepted. A micropolar continuum is a collection of interconnected particles in the form of small rigid bodies undergoing both translational and rotational motions. Different authors, Cheng and He (1995, 1997); Eringen and Suhubi (1964); Eringen (1968); Kumar and Singh (2000), Nappa (1996) and Singh and Kumar (1998a,b), Suhubi and Eringen (1964) and Tomar and Kumar (1999) discussed different types of problems in micropolar elastic medium.

Eringen (1967) extended the theory of micropolar elasticity to obtain linear constitutive theory for micropolar material possessing internal friction. A problem on micropolar viscoelastic waves has been discussed by McCarthy and Eringen (1969). They discussed the propagation conditions and growth equations governing the propagation of waves in micropolar viscoelastic medium.
Cicco and Nappa (1998) discussed a problem on Saint Venant's principle for micropolar viscoelastic bodies. Kumar et al (1990) studied Lamb's plane problem in a micropolar viscoelastic half-space with stretch. Recently, Kumar (2000) discussed wave propagation in micropolar viscoelastic generalized thermoelastic solids. However, most of the problems studied so far, in micropolar viscoelasticity, involves the use of potential functions. The eigen value approach has not been applied in micropolar viscoelastic medium. Mahalanabis and Manna (1989) applied eigen value approach to linear micropolar elasticity by arranging basic equations of linear micropolar elasticity in the form of matrix differential equation. Recently, Mahalanabis and Manna (1997) discussed the problem of linear micropolar thermoelasticity by using the eigen value approach.

In this paper, we consider a two dimensional plane strain problem in a homogeneous isotropic micropolar viscoelastic medium. The solutions were obtained by using eigen value approach after employing integral transformation technique. The integral transforms were inverted using a numerical approach.

\section{Basic equations}

Following Eringen (1967) the constitutive relations and the field equations in micropolar viscoelastic

Keywords. Micropolar; viscoelasticity; eigen value; Laplace and Fourier transforms. 
solid without body forces and body couples can be written as:

$$
\begin{aligned}
t_{k l}= & \lambda_{I} u_{r, r} \delta_{k l}+\mu_{I}\left(u_{k, l}+u_{l, k}\right)+K_{I}\left(u_{l, k}\right. \\
& \left.-\epsilon_{k l r} \phi_{r}\right), \\
m_{k l}= & \alpha_{I} \phi_{r, r} \delta_{k l}+\beta_{I} \phi_{k, l}+\gamma_{I} \phi_{l, k}, \\
\left(\lambda_{I}+\right. & \left.2 \mu_{I}+K_{I}\right) \nabla \nabla \cdot \vec{u}-\left(\mu_{I}+K_{I}\right) \nabla \times \nabla \times \vec{u} \\
+ & K_{I} \nabla \times \vec{\phi}=\rho \frac{\partial^{2} \vec{u}}{\partial t^{2}}, \\
\left(\alpha_{I}+\right. & \left.\beta_{I}+\gamma_{I}\right) \nabla \nabla \cdot \vec{\phi}-\gamma_{I} \nabla \times \nabla \times \vec{\phi}+K_{I} \nabla \times \vec{u} \\
- & 2 K_{I} \vec{\phi}=\rho j \frac{\partial^{2} \vec{\phi}}{\partial t^{2}},
\end{aligned}
$$

where

$$
\begin{gathered}
\lambda_{I}=\lambda+\lambda_{\nu} \frac{\partial}{\partial t}, \quad \mu_{I}=\mu+\mu_{\nu} \frac{\partial}{\partial t}, \\
K_{I}=K+K_{\nu} \frac{\partial}{\partial t}, \\
\alpha_{I}=\alpha+\alpha_{\nu} \frac{\partial}{\partial t}, \quad \beta_{I}=\beta+\beta_{\nu} \frac{\partial}{\partial t}, \\
\gamma_{I}=\gamma+\gamma_{\nu} \frac{\partial}{\partial t},
\end{gathered}
$$

where, $\quad \lambda, \mu, K, \alpha, \beta, \gamma, \lambda_{\nu}, \mu_{\nu}, K_{\nu}, \alpha_{\nu}, \beta_{\nu}, \gamma_{\nu}, \quad$ are material constants, $\rho$ the density, $j$ the micro inertia, $\vec{u}$ the displacement vector, $\vec{\phi}$ the rotation vector, $t_{k l}$ the force stress tensor, $m_{k l}$ the couple stress tensor.

The necessary and sufficient conditions for the internal energy to be non-negative, as given by Eringen (1967) are

$$
\begin{array}{lll}
0 \leq 3 \lambda+2 \mu+K, & 0 \leq \mu, & 0 \leq K, \\
0 \leq 3 \alpha+2 \gamma, & -\gamma \leq \beta \leq \gamma, & 0 \leq \gamma
\end{array}
$$

and

$$
\begin{aligned}
& 0 \leq 3 \lambda_{\nu}+2 \mu_{\nu}+K_{\nu}, \quad 0 \leq \mu_{\nu}, \quad 0 \leq K_{\nu} \\
& 0 \leq 3 \alpha_{\nu}+2 \beta_{\nu}, \quad-\gamma_{\nu} \leq \beta_{\nu} \leq \gamma_{\nu}, 0 \leq \gamma_{\nu} .
\end{aligned}
$$

\section{Formulation and solution}

We consider a homogeneous, isotropic micropolar viscoelastic medium of infinite extent with Cartesian co-ordinates system $(x, y, z)$. To analyze displacement and stresses at the interior of the medium due to concentrated load, the continuum is divided into two half-spaces defined by

- half-space I $|x|<\infty, \quad-\infty<z \leq 0$,

- half-space II $|x|<\infty, 0 \leq z<\infty$.

Since we are discussing a two-dimensional problem, we have

$$
\vec{u}=\left(u_{1}, 0, u_{3}\right), \quad \vec{\phi}=\left(0, \phi_{2}, 0\right) .
$$

Using equation (7), the set of equations (3) and (4) reduce to

$$
\begin{aligned}
\left(\lambda_{I}\right. & \left.+\mu_{I}\right)\left[\frac{\partial^{2} u_{1}}{\partial x^{2}}+\frac{\partial^{2} u_{3}}{\partial x \partial z}\right]+\left(\mu_{I}+K_{I}\right) \\
& \times\left[\frac{\partial^{2} u_{1}}{\partial x^{2}}+\frac{\partial^{2} u_{1}}{\partial z^{2}}\right]-K_{I} \frac{\partial \phi_{2}}{\partial z}=\rho \frac{\partial^{2} u_{1}}{\partial t^{2}}, \\
\left(\lambda_{I}\right. & \left.+\mu_{I}\right)\left[\frac{\partial^{2} u_{1}}{\partial x \partial z}+\frac{\partial^{2} u_{3}}{\partial z^{2}}\right]+\left(\mu_{I}+K_{I}\right) \\
& \times\left[\frac{\partial^{2} u_{3}}{\partial x^{2}}+\frac{\partial^{2} u_{3}}{\partial z^{2}}\right]+K_{I} \frac{\partial \phi_{2}}{\partial x}=\rho \frac{\partial^{2} u_{3}}{\partial t^{2}}, \\
\gamma_{I} & {\left[\frac{\partial^{2} \phi_{2}}{\partial x^{2}}+\frac{\partial^{2} \phi_{2}}{\partial z^{2}}\right]-2 K_{I} \phi_{2}+K_{I}\left[\frac{\partial u_{1}}{\partial z}-\frac{\partial u_{3}}{\partial x}\right] } \\
& =\rho j \frac{\partial^{2} \phi_{2}}{\partial t^{2}} .
\end{aligned}
$$

Introducing the dimensionless quantities

$$
\begin{aligned}
& x^{\prime}=\frac{\omega}{c_{1}} x, \quad z^{\prime}=\frac{\omega}{c_{1}} z, u_{i}^{\prime}=\frac{\omega}{c_{1}} u_{i}, \\
& \phi_{2}^{\prime}=\frac{\mu_{I}}{\rho \omega^{*^{2} j}} \phi_{2}, \quad t^{\prime}=\omega t, \quad t_{i j}^{\prime}=\frac{1}{\mu_{I}} t_{i j}, \\
& m_{i j}^{\prime}=\frac{c_{1}}{\gamma_{I} \omega} m_{i j}, \quad \omega^{* 2}=\frac{K_{I}}{\rho j}, \quad c_{1}^{2}=\frac{\lambda_{I}+2 \mu_{I}+K_{I}}{\rho},
\end{aligned}
$$

where, $\omega$ is the angular frequency.

The equation (8)-(10) reduce to (on suppressing the dashes)

$$
\begin{aligned}
\frac{\partial^{2} u_{1}}{\partial x^{2}}+ & \left(1-a^{2}\right) \frac{\partial^{2} u_{3}}{\partial x \partial z}+a^{2} \frac{\partial^{2} u_{1}}{\partial z^{2}}-s_{4}^{*} \frac{\partial \phi_{2}}{\partial z} \\
= & \frac{1}{\left(s_{1}+s_{2}\right)} \frac{\partial^{2} u_{1}}{\partial t^{2}},
\end{aligned}
$$

$\frac{\partial^{2} u_{3}}{\partial z^{2}}+\left(1-a^{2}\right) \frac{\partial^{2} u_{1}}{\partial x \partial z}+a^{2} \frac{\partial^{2} u_{3}}{\partial x^{2}}+s_{4}^{*} \frac{\partial \phi_{2}}{\partial x}$

$$
=\frac{1}{\left(s_{1}+s_{2}\right)} \frac{\partial^{2} u_{3}}{\partial t^{2}},
$$

$$
\begin{gathered}
\frac{\partial^{2} \phi_{2}}{\partial x^{2}}+\frac{\partial^{2} \phi_{2}}{\partial z^{2}}-\frac{2 c_{1}^{2} K_{I}}{\omega^{2} \gamma_{I}} \phi_{2}+\frac{c_{1}^{2} \mu_{I}}{\omega^{2} \gamma_{I}}\left(\frac{\partial u_{1}}{\partial z}-\frac{\partial u_{3}}{\partial x}\right) \\
=\frac{1}{s_{4}} \frac{\partial^{2} \phi_{2}}{\partial t^{2}}
\end{gathered}
$$

where

$$
\begin{aligned}
& s_{1}=\frac{\left(\lambda_{I}+\mu_{I}\right)}{\rho c_{1}^{2}}, s_{2}=\frac{\left(K_{I}+\mu_{I}\right)}{\rho c_{1}^{2}}, s_{3}=\frac{K_{I} \omega^{*^{2} j}}{\mu_{I} c_{1}^{2}}, \\
& s_{4}=\frac{\gamma_{I}}{\rho c_{1}^{2} j}, \quad a^{2}=\frac{s_{2}}{\left(s_{1}+s_{2}\right)}, s_{4}^{*}=\frac{s_{3}}{\left(s_{1}+s_{2}\right)} .
\end{aligned}
$$


Applying Laplace transform w.r.t time ' $t$ ' defined by

$$
\begin{gathered}
\left\{\bar{u}_{i}(x, z, p), \bar{\phi}_{2}(x, z, p)\right\}=\int_{0}^{\infty}\left\{u_{i}(x, z, t),\right. \\
\left.\phi_{2}(x, z, t)\right\} e^{-p t} d t
\end{gathered}
$$

and then Fourier transform w.r.t ' $x$ ' defined by

$$
\begin{gathered}
\left\{\tilde{u}_{i}(\xi, z, p), \tilde{\phi}_{2}(\xi, z, p)\right\}=\int_{-\infty}^{\infty}\left\{\bar{u}_{i}(x, z, p), \bar{\phi}_{2}\right. \\
(x, z, p)\} e^{\iota \xi x} d x, i=1,3 .
\end{gathered}
$$

on equations (11)-(13), we obtain

$$
\begin{aligned}
\tilde{u}_{1}^{\prime \prime}= & \frac{1}{a^{2}}\left[\xi^{2}+\frac{p^{2}}{\left(s_{1}+s_{2}\right)}\right] \tilde{u}_{1}+\frac{\iota \xi\left(1-a^{2}\right)}{a^{2}} \tilde{u}_{3}^{\prime} \\
& +\frac{s_{4}^{*}}{a^{2}} \tilde{\phi}_{2}^{\prime} \\
\tilde{u}_{3}^{\prime \prime}= & {\left[a^{2} \xi^{2}+\frac{p^{2}}{\left(s_{1}+s_{2}\right)}\right] \tilde{u}_{3}+\iota \xi s_{4}^{*} \tilde{\phi}_{2}+\iota \xi } \\
& \left(1-a^{2}\right) \tilde{u}_{1}^{\prime},
\end{aligned}
$$

and

$$
\begin{aligned}
\tilde{\phi}^{\prime \prime}{ }_{2}= & -\frac{c_{1}^{2} \mu_{I}}{\omega^{2} \gamma_{I}} \tilde{u}_{1}^{\prime}-\frac{\iota \xi c_{1}^{2} \mu_{I}}{\omega^{2} \gamma_{I}} \tilde{u}_{3}+ \\
& {\left[\xi^{2}+\frac{2 c_{1}^{2} K_{I}}{\omega^{2} \gamma_{I}}+\frac{p^{2}}{s_{4}}\right] \tilde{\phi}_{2} . }
\end{aligned}
$$

The system of equations (17)-(19) can be written as

$$
\frac{d}{d z} W(\xi, z, p)=A(\xi, p) W(\xi, z, p),
$$

where

$$
\begin{aligned}
W= & {\left[\begin{array}{c}
U \\
U^{\prime}
\end{array}\right], \quad A=\left[\begin{array}{cc}
O & I \\
A_{2} & A_{1}
\end{array}\right], U=\left[\begin{array}{l}
\tilde{u}_{1} \\
\tilde{u}_{3} \\
\tilde{\phi}_{2}
\end{array}\right], } \\
O= & {\left[\begin{array}{lll}
0 & 0 & 0 \\
0 & 0 & 0 \\
0 & 0 & 0
\end{array}\right], I=\left[\begin{array}{lll}
1 & 0 & 0 \\
0 & 1 & 0 \\
0 & 0 & 1
\end{array}\right], } \\
A_{1}= & {\left[\begin{array}{lll}
0 & \frac{\iota \xi\left(1-a^{2}\right)}{a^{2}} & \frac{s_{4}^{*}}{a^{2}} \\
\iota \xi\left(1-a^{2}\right) & 0 & 0 \\
\frac{-c_{1}^{2} \mu_{I}}{\omega^{2} \gamma_{I}} & 0 & 0
\end{array}\right], } \\
A_{2}= & {\left[\begin{array}{cc}
\frac{1}{a^{2}}\left(\xi^{2}+\frac{p^{2}}{\left(s_{1}+s_{2}\right)}\right) & 0 \\
0 & a^{2} \xi^{2}+\frac{p^{2}}{\left(s_{1}+s_{2}\right)} \\
0 & \frac{-\iota \xi c_{1}^{2} \mu_{I}}{\omega^{2} \gamma_{I}}
\end{array}\right.} \\
& \left.\times \quad \begin{array}{c}
\iota \xi s_{4}^{*} \\
\xi^{2}+\frac{2 c_{1}^{2} K_{I}}{\omega^{2} \gamma_{I}}+\frac{p^{2}}{s_{4}}
\end{array}\right] .
\end{aligned}
$$

To solve equation (20), we take

$$
W(\xi, z, p)=X(\xi, p) e^{q z}
$$

so that

$$
A(\xi, p) W(\xi, z, p)=q W(\xi, z, p)
$$

which leads to eigen value problem. The characteristic equation corresponding to the matrix $A$ is given by

$$
\operatorname{det}[A-q I]=0
$$

which on expansion provides us

$$
q^{6}-\lambda_{1} q^{4}+\lambda_{2} q^{2}-\lambda_{3}=0
$$

where,

$$
\begin{aligned}
\lambda_{1}= & \left(1+\frac{1}{a^{2}}\right) \frac{p^{2}}{\left(s_{1}+s_{2}\right)}+ \\
& {\left[3 \xi^{2}+\frac{2 c_{1}^{2} K_{I}}{\omega^{2} \gamma_{I}}+\frac{p^{2}}{s_{4}}-\frac{c_{1}^{2} \mu_{I} s_{4}^{*}}{\omega^{2} \gamma_{I} a^{2}}\right] } \\
\lambda_{2}= & {\left[\xi^{2}+\frac{2 c_{1}^{2} K_{I}}{\omega^{2} \gamma_{I}}+\frac{p^{2}}{s_{4}}\right]\left[\frac{p^{2}}{\left(s_{1}+s_{2}\right)}\left(1+\frac{1}{a^{2}}\right)\right.} \\
& \left.+2 \xi^{2}\right]-\frac{c_{1}^{2} \mu_{I} s_{4}^{*}}{\omega^{2} \gamma_{I} a^{2}}\left[2 \xi^{2}+\frac{p^{2}}{\left(s_{1}+s_{2}\right)}\right]+\frac{1}{a^{2}} \\
& {\left[\xi^{2}+\frac{p^{2}}{\left(s_{1}+s_{2}\right)}\right]\left[a^{2} \xi^{2}+\frac{p^{2}}{\left(s_{1}+s_{2}\right)}\right], }
\end{aligned}
$$

and

$$
\begin{aligned}
\lambda_{3}= & \frac{1}{a^{2}}\left[\xi^{2}+\frac{p^{2}}{\left(s_{1}+s_{2}\right)}\right]\left[a^{2} \xi^{2}+\frac{p^{2}}{\left(s_{1}+s_{2}\right)}\right] \\
& {\left[\xi^{2}+\frac{2 c_{1}^{2} K_{I}}{\omega^{2} \gamma_{I}}+\frac{p^{2}}{s_{4}}\right]-\frac{s_{4}^{*}}{a^{2}}\left[\xi^{2}+\frac{p^{2}}{\left(s_{1}+s_{2}\right)}\right] } \\
& \xi^{2} \frac{c_{1}^{2} \mu_{I}}{\omega^{2} \gamma_{I}} .
\end{aligned}
$$

The roots of equation (25) are $\pm q_{i}, \quad i=1,2,3$.

The eigen values of the matrix $A$ are the roots of equation (25). We assume that real parts of $q_{i}$ are positive. The vector $X(\xi, p)$ corresponding to the eigen values $q_{i}$ can be determined by solving the homogeneous equation

$$
[A-q I] X(\xi, p)=0 .
$$

The set of eigen vectors $X_{i}(\xi, p),(i=1,2,3,4,5,6)$ may be obtained as

$$
X_{i}(\xi, p)=\left[\begin{array}{c}
X_{i 1}(\xi, p) \\
X_{i 2}(\xi, p)
\end{array}\right]
$$


where

$$
\begin{gathered}
X_{i 1}(\xi, p)=\left[\begin{array}{c}
a_{i} q_{i} \\
b_{i} \\
-\xi
\end{array}\right], \quad X_{i 2}(\xi, p)=\left[\begin{array}{c}
a_{i} q_{i}^{2} \\
b_{i} q_{i} \\
-\xi q_{i}
\end{array}\right], \\
X_{j 1}(\xi, p)=\left[\begin{array}{c}
-a_{i} q_{i} \\
b_{i} \\
-\xi
\end{array}\right], \quad X_{j 2}(\xi, p)=\left[\begin{array}{c}
a_{i} q_{i}^{2} \\
-b_{i} q_{i} \\
\xi q_{i}
\end{array}\right], \\
j=i+3, q=-q_{i} ; i=1,2,3 \quad(32) \\
a_{i}=\left[\xi\left(a^{2}-1\right)\left\{\left(\xi^{2}+\frac{2 c_{1}^{2} K_{I}}{\omega^{2} \gamma_{I}}+\frac{p^{2}}{s_{4}}\right)-q_{i}^{2}\right\}\right. \\
\left.-\frac{c_{1}^{2} \mu_{I} s_{4}^{*} \xi}{\omega^{2} \gamma_{I}}\right] / \Delta_{i}, \quad(33) \\
b_{i}=-\iota\left[\left(\xi^{2}+\frac{p^{2}}{\left(s_{1}+s_{2}\right)}\right)\left(\xi^{2}+\frac{2 c_{1}^{2} K_{I}}{\omega^{2} \gamma_{I}}+\frac{p^{2}}{s_{4}}\right)\right. \\
+a^{2} q_{i}^{2}\left\{q_{i}^{2}-\left(\xi^{2}+\frac{2 c_{1}^{2} K_{I}}{\omega^{2} \gamma_{I}}+\frac{p^{2}}{s_{4}}\right)\right\}-\left(\xi^{2}\right. \\
\left.\left.+\frac{p^{2}}{\left(s_{1}+s_{2}\right)}\right) q_{i}^{2}+\frac{c_{1}^{2} \mu_{I} s_{4}^{*} q_{i}^{2}}{\omega^{2} \gamma_{I}}\right] / \Delta_{i}, \\
\left.\left.\Delta_{i}=\frac{c_{1}^{2} \mu_{I}\left[q_{i}^{2}-\left(\xi^{2}+\frac{p^{2}}{\omega^{2} \gamma_{I}}\right.\right.}{\left(s_{1}+s_{2}\right)}\right)\right],
\end{gathered}
$$

The solution of equation (20) is given by

$$
\begin{aligned}
W(\xi, z, p)= & \sum_{i=1}^{3}\left[B_{i} X_{i}(\xi, p) \exp \left(q_{i} z\right)+B_{i+3}\right. \\
& \left.X_{i+3}(\xi, p) \exp \left(-q_{i} z\right)\right]
\end{aligned}
$$

where, $B_{i}(i=1,2,3,4,5,6)$ are arbitrary constants.

The equation (36) represents the solution of the general problem in the plane strain case of micropolar viscoelasticity by employing the eigenvalue approach and therefore can be applied to a broad class of problem in the domains of Laplace and Fourier transforms.

\section{Application}

We consider an infinite micropolar viscoelastic space in which a concentrated force of magnitude $F=-F_{o} \delta(x) \delta(t)$, acting in the direction of the $z$ axis at the origin of the Cartesian co-ordinate system. The problem is plane strain w.r.t the $z$-axis and the boundary conditions at the interface of two half-spaces $(z=0)$ are given by

$$
\begin{aligned}
& u_{1}\left(x, 0^{+}, t\right)-u_{1}\left(x, 0^{-}, t\right)=0, \\
& u_{3}\left(x, 0^{+}, t\right)-u_{3}\left(x, 0^{-}, t\right)=0,
\end{aligned}
$$

$$
\begin{aligned}
\phi_{2}\left(x, 0^{+}, t\right)-\phi_{2}\left(x, 0^{-}, t\right) & =0, \\
m_{32}\left(x, 0^{+}, t\right)-m_{32}\left(x, 0^{-}, t\right) & =0, \\
t_{31}\left(x, 0^{+}, t\right)-t_{31}\left(x, 0^{-}, t\right) & =0, \\
t_{33}\left(x, 0^{+}, t\right)-t_{33}\left(x, 0^{-}, t\right) & =-F_{o} \delta(x) \delta(t) .
\end{aligned}
$$

Applying the Laplace and Fourier transforms on equations (37)-(40), we get

$$
\begin{aligned}
\tilde{u}_{1}\left(\xi, 0^{+}, p\right)-\tilde{u}_{1}\left(\xi, 0^{-}, p\right) & =0, \\
\tilde{u}_{3}\left(\xi, 0^{+}, p\right)-\tilde{u}_{3}\left(\xi, 0^{-}, p\right) & =0, \\
\tilde{\phi}_{2}\left(\xi, 0^{+}, p\right)-\tilde{\phi}_{2}\left(\xi, 0^{-}, p\right) & =0, \\
\tilde{m}_{32}\left(\xi, 0^{+}, p\right)-\tilde{m}_{32}\left(\xi, 0^{-}, p\right) & =0, \\
\tilde{t}_{31}\left(\xi, 0^{+}, p\right)-\tilde{t}_{31}\left(\xi, 0^{-}, p\right) & =0, \\
\tilde{t}_{33}\left(\xi, 0^{+}, p\right)-\tilde{t}_{33}\left(\xi, 0^{-}, p\right) & =F_{o} .
\end{aligned}
$$

The transformed displacement, microrotation and stresses are given for $z \geq 0$ as

$$
\begin{aligned}
\tilde{u}_{1}(\xi, z, p)= & -\left\{a_{1} q_{1} B_{4} \exp \left(-q_{1} z\right)+a_{2} q_{2} B_{5} \exp \right. \\
& \left.\left(-q_{2} z\right)+a_{3} q_{3} B_{6} \exp \left(-q_{3} z\right)\right\}, \quad(45) \\
\tilde{u}_{3}(\xi, z, p)= & b_{1} B_{4} \exp \left(-q_{1} z\right)+b_{2} B_{5} \exp \left(-q_{2} z\right) \\
& +b_{3} B_{6} \exp \left(-q_{3} z\right), \\
\tilde{\phi}_{2}(\xi, z, p)= & -\xi\left\{B_{4} \exp \left(-q_{1} z\right)+B_{5} \exp \left(-q_{2} z\right)\right. \\
& \left.+B_{6} \exp \left(-q_{3} z\right)\right\}, \\
\tilde{m}_{32}(\xi, z, p)= & s_{7} \xi\left\{q_{1} B_{4} \exp \left(-q_{1} z\right)+q_{2} B_{5} \exp \right. \\
& \left.\left(-q_{2} z\right)+q_{3} B_{6} \exp \left(-q_{3} z\right)\right\}, \\
\tilde{t}_{31}(\xi, z, p)=\{ & \left.a_{1} q_{1}^{2} s_{8}-\iota \xi b_{1}+\xi s_{9}\right\} B_{4} \exp \left(-q_{1} z\right) \\
+ & \left\{a_{2} q_{2}^{2} s_{8}-\iota \xi b_{2}+\xi s_{9}\right\} B_{5} \exp \left(-q_{2} z\right) \\
+ & \left\{a_{3} q_{3}^{2} s_{8}-\iota \xi b_{3}+\xi s_{9}\right\} B_{6} \exp \left(-q_{3} z\right),
\end{aligned}
$$

$$
\begin{aligned}
\tilde{t}_{33}(\xi, z, p)= & -\left[q_{1}\left(b_{1} s_{6}-\iota \xi a_{1} s_{5}\right) B_{4} \exp \left(-q_{1} z\right)\right. \\
& +q_{2}\left(b_{2} s_{6}-\iota \xi a_{2} s_{5}\right) B_{5} \exp \left(-q_{2} z\right)+q_{3} \\
& \left.\times\left(b_{3} s_{6}-\iota \xi a_{3} s_{5}\right) B_{6} \exp \left(-q_{3} z\right)\right], \quad(50)
\end{aligned}
$$

and for $z \leq 0$ as

$$
\begin{aligned}
\tilde{u}_{1}(\xi, z, p)= & a_{1} q_{1} B_{1} \exp \left(q_{1} z\right)+a_{2} q_{2} B_{2} \exp \left(q_{2} z\right) \\
& +a_{3} q_{3} B_{3} \exp \left(q_{3} z\right), \\
\tilde{u}_{3}(\xi, z, p)= & b_{1} B_{1} \exp \left(q_{1} z\right)+b_{2} B_{2} \exp \left(q_{2} z\right) \\
& +b_{3} B_{3} \exp \left(q_{3} z\right), \\
\tilde{\phi}_{2}(\xi, z, p)= & -\xi\left\{B_{1} \exp \left(q_{1} z\right)+B_{2} \exp \left(q_{2} z\right)\right. \\
& \left.+B_{3} \exp \left(q_{3} z\right)\right\}, \\
\tilde{m}_{32}(\xi, z, p)= & -s_{7} \xi\left\{q_{1} B_{1} \exp \left(q_{1} z\right)+q_{2} B_{2} \exp \right. \\
& \left.\left(q_{2} z\right)+q_{3} B_{3} \exp \left(q_{3} z\right)\right\}, \\
\tilde{t}_{31}(\xi, z, p)= & \left\{a_{1} q_{1}^{2} s_{8}-\iota \xi b_{1}+\xi s_{9}\right\} B_{1} \exp \left(q_{1} z\right) \\
& +\left\{a_{2} q_{2}^{2} s_{8}-\iota \xi b_{2}+\xi s_{9}\right\} B_{2} \exp \left(q_{2} z\right) \\
& +\left\{a_{3} q_{3}^{2} s_{8}-\iota \xi b_{3}+\xi s_{9}\right\} B_{3} \exp \left(q_{3} z\right),
\end{aligned}
$$




$$
\begin{aligned}
\tilde{t}_{33}(\xi, z, p)= & {\left[q_{1}\left(b_{1} s_{6}-\iota \xi a_{1} s_{5}\right) B_{1} \exp \left(q_{1} z\right)+q_{2}\right.} \\
& \left(b_{2} s_{6}-\iota \xi a_{2} s_{5}\right) B_{2} \exp \left(q_{2} z\right)+q_{3}\left(b_{3}\right. \\
& \left.\left.s_{6}-\iota \xi a_{3} s_{5}\right) B_{3} \exp \left(q_{3} z\right)\right],
\end{aligned}
$$

where

$$
\begin{aligned}
& s_{5}=\frac{\lambda_{I}}{\mu_{I}}, \quad s_{6}=\frac{\left(\lambda_{I}+2 \mu_{I}+K_{I}\right)}{\mu_{I}}, s_{7}=\frac{\rho \omega^{*^{2}} j}{\mu_{I}}, \\
& s_{8}=\frac{\mu_{I}+K_{I}}{\mu_{I}}, s_{9}=\frac{K_{I} \rho \omega^{*^{2}} j}{\mu_{I}^{2}}
\end{aligned}
$$

Using conditions (41)-(44) in equations (45)-(56), we obtain

$$
\begin{aligned}
& a_{1} q_{1}\left(B_{1}+B_{4}\right)+a_{2} q_{2}\left(B_{2}+B_{5}\right)+a_{3} q_{3} \\
& \left(B_{3}+B_{6}\right)=0 \text {, } \\
& \left(B_{1}-B_{4}\right)+\left(B_{2}-B_{5}\right)+\left(B_{3}-B_{6}\right)=0 \text {, } \\
& b_{1}\left(B_{1}-B_{4}\right)+b_{2}\left(B_{2}-B_{5}\right)+b_{3}\left(B_{3}-B_{6}\right)=0 \text {, } \\
& q_{1}\left(B_{1}+B_{4}\right)+q_{2}\left(B_{2}+B_{5}\right)+q_{3}\left(B_{3}+B_{6}\right)=0 \text {, } \\
& {\left[a_{1} q_{1}^{2} s_{8}-\iota \xi b_{1}+\xi s_{9}\right]\left(B_{1}-B_{4}\right)+\left[a_{2} q_{2}^{2} s_{8}-\iota \xi b_{2}\right.} \\
& \left.+\xi s_{9}\right]\left(B_{2}-B_{5}\right)+\left[a_{3} q_{3}^{2} s_{8}-\iota \xi b_{3}+\xi s_{9}\right] \\
& \left(B_{3}-B_{6}\right)=0 \text {, } \\
& q_{1}\left(b_{1} s_{6}-\iota \xi a_{1} s_{5}\right)\left(B_{1}+B_{4}\right)+q_{2}\left(b_{2} s_{6}-\iota \xi a_{2} s_{5}\right) \\
& \left(B_{2}+B_{5}\right)+q_{3}\left(b_{3} s_{6}-\iota \xi a_{3} s_{5}\right)\left(B_{3}+B_{6}\right)=F_{o} \text {. }
\end{aligned}
$$

Solving system of equations (58)-(63), we obtain

$$
\begin{aligned}
& B_{1}=B_{4}=\frac{F_{o}\left(a_{3}-a_{2}\right)}{q_{1} \Delta}, \\
& B_{2}=B_{5}=\frac{F_{o}\left(a_{1}-a_{3}\right)}{q_{2} \Delta}, \\
& B_{3}=B_{6}=\frac{F_{o}\left(a_{2}-a_{1}\right)}{q_{3} \Delta},
\end{aligned}
$$

where

$$
\begin{aligned}
\Delta= & 2 s_{6}\left[\left(a_{2} b_{3}-a_{3} b_{2}\right)+\left(a_{3} b_{1}-a_{1} b_{3}\right)\right. \\
& \left.+\left(a_{1} b_{2}-a_{2} b_{1}\right)\right] .
\end{aligned}
$$

Thus the functions $\tilde{u}_{1}, \tilde{u}_{3}, \tilde{\phi}_{2}, \tilde{m}_{32}, \tilde{t}_{31}$ and $\tilde{t}_{33}$ have been determined in the transformed domain and these enable us to find the displacement, microrotation and stresses.

Particular Case: If we neglect the effect of viscocity, that is, when $\chi_{I}=\chi$ where $\chi=\lambda, \mu, K, \gamma$, we obtain the expressions for displacement component, force stresses and couple stress in micropolar elastic medium.

Sub-Case: Neglecting the effect of micropolarity, the analytical expressions for displacement component and force stresses may be obtained in classical theory of elasticity.

\section{Inversion of transforms}

The transformed displacements and stresses are functions of $z$, the parameters of Laplace and Fourier transforms $p$ and $\xi$ respectively, and hence are of the form $\tilde{f}(\xi, z, p)$. To get the function $f(x, z, t)$ in the physical domain, first we invert the Fourier transform using

$$
\begin{aligned}
\bar{f}(x, z, p) & =\frac{1}{2 \pi} \int_{-\infty}^{\infty} e^{-\iota \xi x} \tilde{f}(\xi, z, p) d \xi \\
& =\frac{1}{\pi} \int_{0}^{\infty}\left\{\cos (\xi x) f_{e}-\iota \sin (\xi x) f_{o}\right\} d \xi
\end{aligned}
$$

where $f_{e}$ and $f_{o}$ are even and odd parts of the function $\tilde{f}(\xi, z, p)$ respectively. Thus, expression (68) gives us the Laplace $\bar{f}(x, z, p)$ of the function $f(x, z, t)$.

Now, for the fixed values of $\xi, x$ and $z$, the $\bar{f}(x, z, p)$ in the expression (68) can be considered as the Laplace transform $\bar{g}(p)$ of some function $g(t)$. Following Honig and Hirdes (1984), the Laplace transformed function $\bar{g}(p)$ can be inverted as given below.

The function $g(t)$ can be obtained by using

$$
g(t)=\frac{1}{2 \pi \iota} \int_{C-\iota \infty}^{C+\iota \infty} e^{p t} \bar{g}(p) d p,
$$

where $C$ is an arbitrary real number greater than all the real parts of the singularities of $\bar{g}(p)$. Taking $p=C+\iota y$, we get

$$
g(t)=\frac{e^{C t}}{2 \pi} \int_{-\infty}^{\infty} e^{\iota t y} \bar{g}(C+\iota y) d y
$$

Now, taking $e^{-C t} g(t)$ as $h(t)$ and expanding it as Fourier series in $[0,2 L]$, we obtain approximately the formula

$$
g(t)=g_{\infty}(t)+E_{D^{\prime}}
$$

where

$$
\begin{aligned}
g_{\infty}(t) & =\frac{C_{o}}{2}+\sum_{k=1}^{\infty} C_{k}, \quad 0 \leq t \leq 2 L, \\
C_{k} & =\frac{e^{C t}}{L} \operatorname{Re}\left[e^{\frac{\iota k \pi t}{L}} \bar{g}\left(C+\frac{\iota k \pi}{L}\right)\right],
\end{aligned}
$$

$E_{D}$ is the discretization error and can be made arbitrarily small by choosing $C$ large enough. The value of $C$ and $L$ are chosen according to the criteria outlined by Honig and Hirdes (1984). 


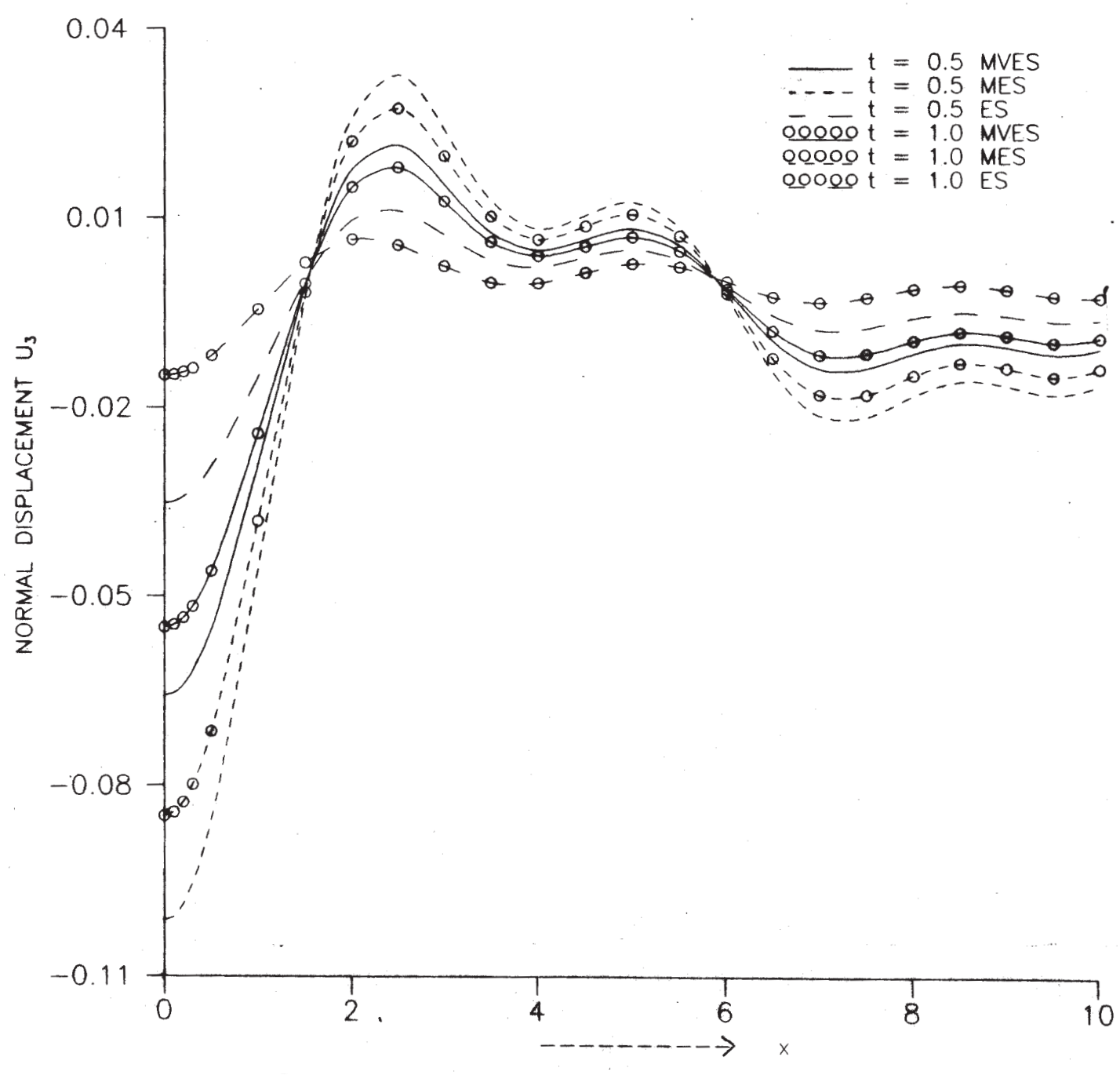

Figure 1. Variation of normal displacement $U_{3}(x, 1), U_{3}=u_{3} / F_{0}$ with distance $x$.

Since the infinite series in equation $(72)$ can be summed up only to a finite number of $N$ terms, so the approximate value of $g(t)$ becomes

$$
g_{N}(t)=\frac{C_{o}}{2}+\sum_{k=1}^{N} C_{k}, \quad 0 \leq t \leq 2 L .
$$

Now, we introduce a truncation error $E_{T}$ that must be added to the discretization error to produce the total approximation error in evaluating $g(t)$ using the above formula. Two methods are used to reduce the total error. The discretization error is reduced by using the 'Korrecktur' method, Honig and Hirdes (1984) and then ' $\epsilon$-algorithm' is used to reduce the truncation error and hence to accelerate the convergence.

The 'Korrecktur'- method formula, to evaluate the function $g(t)$ is

$$
g(t)=g_{\infty}(t)-e^{-2 C L} g_{\infty}(2 L+t)+E_{D}^{\prime},
$$

where

$$
\left|E_{D}^{\prime}\right| \ll\left|E_{D}\right| .
$$

Thus, the approximate value of $g(t)$ becomes

$$
g_{N_{k}}(t)=g_{N}(t)-e^{-2 C L} g_{N^{\prime}}^{(2 L+t)},
$$

where, $N^{\prime}$ is an integer such that $N^{\prime}<N$.

We shall now describe the $\epsilon$-algorithm which is used to accelerate the convergence of the series in equation (73). Let $N$ be a natural number and $S_{m}=\sum_{k=1}^{m} C_{k}$ be the sequence of partial sums of equation (73). We define the $\epsilon$-sequence by

$$
\begin{aligned}
& \epsilon_{0, m}=0, \quad \epsilon_{1, m}=S_{m}, \\
& \epsilon_{n+1, m}=\epsilon_{n-1, m+1}+\frac{1}{\epsilon_{n, m+1}-\epsilon_{n, m}} \\
& n, m=1,2,3, \ldots . .
\end{aligned}
$$

It can be shown (Honig and Hirdes 1984) that the sequence $\epsilon_{1,1}, \epsilon_{3,1}, \ldots, \epsilon_{N, 1}$ converges to $g(t)+$ 


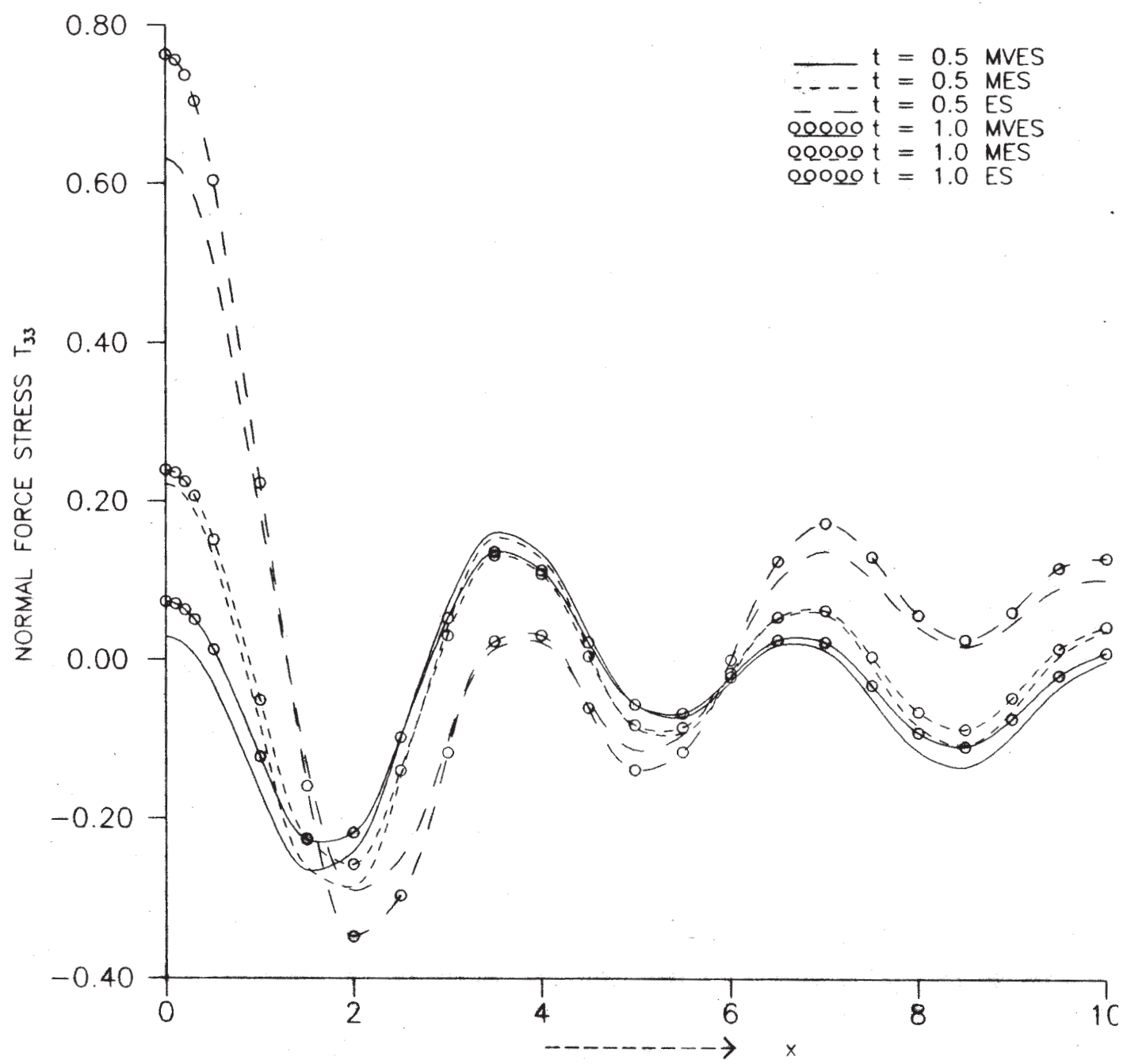

Figure 2. Variation of normal force stress $T_{33}(x, 1), T_{33}=t_{33} / F_{o}$ with distance $x$.

$E_{D}-C_{o} / 2$ faster than the sequence of partial $S_{m}, m=1,2,3, \ldots$. The actual procedure to invert the Laplace transform consists of equation (75) together with the $\epsilon$-algorithm.

The last step is to evaluate the integral in equation (68). The method for evaluating this integral by Press et al (1986), which involves the use of Romberg's integration with adaptive step size. This, also uses the results from successive refinement of the extended trapezoidal rule followed by extrapolation of the results to the limit when the step size tends to zero.

\section{Numerical results and discussion}

Following Gauthier (1982), we take the following values of relevant parameters for the case of Aluminum epoxy composite as

$$
\begin{aligned}
\rho=2.19 \mathrm{gm} / \mathrm{cm}^{3}, \quad \lambda= & 7.59 \times \\
& 10^{10} \text { dyne } / \mathrm{cm}^{2},
\end{aligned}
$$

$$
\begin{aligned}
\mu=1.89 \times 10^{10} \text { dyne } / \mathrm{cm}^{2}, \quad K= & 0.0149 \times \\
& 10^{10} \mathrm{dyne} / \mathrm{cm}^{2}, \\
\gamma=0.0268 \times 10^{10} \text { dyne }, \quad j= & 0.00196 \mathrm{~cm}^{2} .
\end{aligned}
$$

For a particular model of micropolar viscoelastic solid the relevant parameters are expressed as

$$
\begin{array}{r}
\chi_{I}=\chi\left(1+\iota Q_{i}^{-1}\right), \quad i=1,2,3,4 \text { for } \chi=\lambda, \mu, \\
K, \gamma \text { respectively }
\end{array}
$$

where

$$
Q_{1}=0.05, \quad Q_{2}=0.1, \quad Q_{3}=0.15, \quad Q_{4}=0.1 .
$$

The comparison of values of normal displacement $U_{3}\left[=u_{3} / F_{o}\right]$, normal force stress $T_{33}[=$ $\left.t_{33} / F_{o}\right]$ and couple stress $M_{32}\left[=m_{32} / F_{o}\right]$, for micropolar viscoelastic solid (MVES), micropolar elastic solid (MES) and elastic solid (ES) have been studied. The computations were carried out for two values of time $t=0.5$ and $t=1.0$ and 


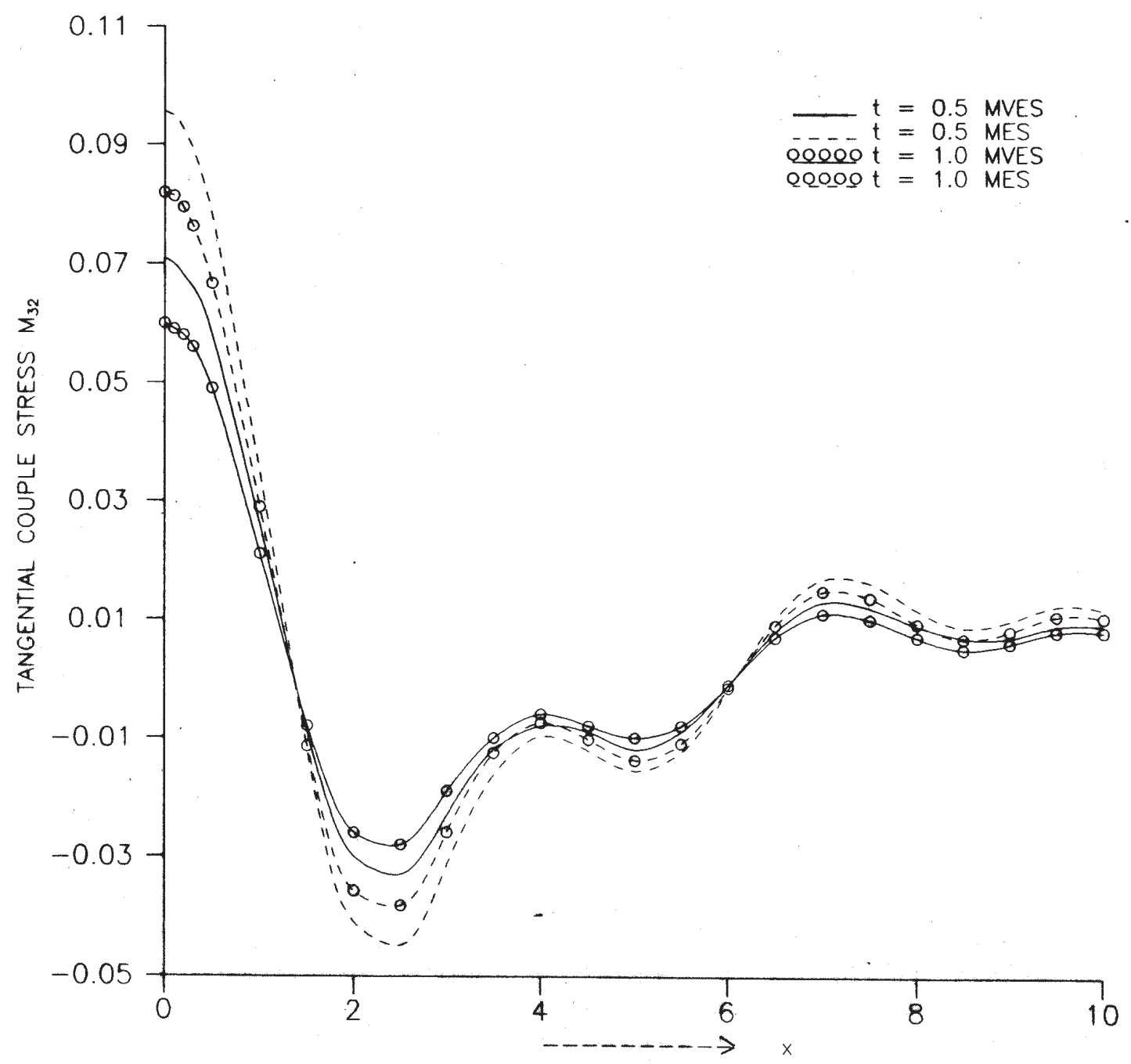

Figure 3. Variation of tangential couple stress $M_{32}(x, 1), M_{32}=m_{32} / F_{o}$ with distance $x$.

for $\omega=1 \times 10^{10} \mathrm{sec}^{-1}$ at $z=1.0$ in the range $0 \leq x \leq 10$. The solid lines [-] in graphs represent the variations for MEVS, the small dashed lines $[-----]$ represent the variations for MES and large dashed lines $[-\ldots+-]$ represent the variations for ES. For all three cases solid or dashed lines with center symbols represent the variations for time $t=1.0$, whereas without center symbols are for time $t=0.5$.

Figure 1 shows the variations of normal displacement $U_{3}$ with $x$. For both the times as well as for all three solids, the values of $U_{3}$ are initially increasing and then start oscillating with the further increase in $x$. Initially, value of $U_{3}$ is smaller for MES and greater for ES than that for MVES. As time increases from $t=0.5$ to $t=1.0$ the values of $U_{3}$ increases in the range $0 \leq x \leq 2$ and $6 \leq x \leq 10$ for all three cases. It is observed that the maximum displacement occur at the maximum time, i.e., $t=1.0$, in response to the source for all three cases.
Figure 2 shows the variations of normal force stress $T_{33}$ with $x$. For all three cases MVES, MES and $\mathrm{ES}$, the values of $T_{33}$ for time $t=0.5$ are less than those for time $t=1.0$ in the ranges $0 \leq x \leq 2$ and $6 \leq x \leq 10$. For fixed time $t=0.5$ the values of $T_{33}$ for MVES are less than those for MES in the range $0 \leq x \leq 2$ and $6 \leq x \leq 10$ whereas for MES, the values are more than those for ES in same ranges. Similar trend is observed for time $t=1.0$. Initially the values decrease sharply and then follow oscillatory pattern with reference to $x$.

Figure 3 shows the variations of couple stress $M_{32}$ with $x$. The values of $M_{32}$ have been taken by multiplying their original values with 10 . With the maximum couple stress at the initial value, i.e., $x=0$ the values of $M_{32}$ decrease sharply for all the two cases of MVES and MES. Further, with the increase in $x$, for a fixed time ' $t$ ', the couple stress $M_{32}$, follows oscillatory behaviour. The value of $M_{32}$ is more for time $t=0.5$ than those for time $t=1.0$ in the ranges $0 \leq x \leq 1.5$ and $6 \leq x \leq 10$ 
for both the cases. For both the times the values for MVES is less than those for MES in the ranges $0 \leq x \leq 1.5$ and $6 \leq x \leq 10$.

\section{Acknowledgement}

One of the authors (Suman Choudhary) is thankful to Kurukshetra University for supporting a financial assistance in the form of a University Research Scholarship.

\section{References}

Cheng Z-Q and He L-H 1995 Micropolar elastic field due to a spherical inclusion; Int. J. Engng. Sci. 33 389-397

Cheng Z-Q and He L-H 1997 Micropolar elastic field due to a circular inclusion; Int. J. Engng. Sci. 35 659-668

Cicco S De and Nappa L 1998 On Saint Venant's principle for micropolar viscoelastic bodies, Int. J. Engng. Sci. 36 883-893

Eringen A C and Suhubi E S 1964 Non-linear theory of simple micropolar solids I; Int. J. Engng. Sci. 2 189-203

Eringen A C 1966a Linear theory of micropolar elasticity; J. Math. Mech. 15 909-924

Eringen A C 1966b Theory of micropolar fluids; J. Math. Mech. 16 1-18

Eringen A C 1967 Linear theory of micropolar viscoelasticity; Int. J. Engng. Sci. 5 191-729

Eringen A C 1968 Theory of micropolar elasticity in fracture Vol II, (Academic Press) 621-729

Eringen A C 1976 Non-local polar field theories. In: Continuum Physics (ed.) A C Eringen, Vol.IV (New York, Academic Press) 205-267

Gauthier R D 1982 In: Experimental investigations on micropolar media, mechanics of micropolar media, (ed.) O Brulin and R K T Hseieh, (Singapore: World Scientific)
Honig G and Hirdes U 1984 A method for the numerical inversion of the Laplace transforms; J. Comp. Appl. Math. 10 113-132

Kumar R, Gogna M L and Lokenath Debnath 1990 On Lamb's plane problem in micropolar viscoelastic halfspace with stretch; Int. J. Math. \& Math. Sci. 132 363372

Kumar R and Singh B 2000 Refelection of plane waves at a planar viscoelastic micropolar interface; Indian J. Pure Appl. Math. 31 287-303

Kumar R 2000 Wave propagation in micropolar viscoelastic generalized thermoelastic solid; Int. J. Engng. Sci. 38 $1377-1395$

Mahalanabis R K and Manna J 1989 Eigenvalue approach to linear micropolar elasticity; Indian J. Pure Appl. Math. 20 1237-1250

Mahalanabis R K and Manna J 1997 Eigenvalue approach to the problem of linear micropolar thermoelasticity; Indian Acad. Math. Sci. 19 69-86

McCarthy M F and Eringen A C 1969 Micropolar viscoelastic waves; Int. J. Engng. Sci. 7 447-458

Nappa L 1996 Decay estimates for micropolar elastic cylinders; Int. J. Engng. Sci. 34 1601-1609

Press W H, Teukolsky S A, Vellerlig W T and Flannery B P 1986 (Second edition): Numerical Recipes in FORTRAN, (Cambridge University Press, Cambridge)

Singh B and Kumar R 1998a Reflection and refraction of plane waves at an interface between micropolar elastic solid and viscoelastic solid; Int. J. Engng. Sci. 36 119135

Singh B and Kumar R 1998b Reflection and refraction of plane waves from flat boundary of micropolar generalized thermoelastic half-space; Int. J. Engng. Sci. 36 865-890

Suhubi E S and Eringen A C 1964 Non-linear theory of micropolar solid II; Int. J. Engng. Sci. 2 389-404

Tomar S K and Kumar R 1999 Elastic wave propagation in a cylindrical bore situated in a micropolar elastic media with stretch; Proc. Indian Acad. Sci. (Math. Sci) $\mathbf{1 8 9}$ 425-435 